\title{
Article \\ A Combined Transcriptomics and Proteomics Approach Reveals the Differences in the Predatory and Defensive Venoms of the Molluscivorous Cone Snail Cylinder ammiralis (Caenogastropoda: Conidae)
}

\author{
Samuel Abalde ${ }^{1,2, *(\mathbb{D})}$, Sébastien Dutertre ${ }^{3}(\mathbb{D})$ and Rafael Zardoya ${ }^{1}$ (D) \\ 1 Departamento de Biodiversidad y Biología Evolutiva, Museo Nacional de Ciencias Naturales (MNCN-CSIC), \\ José Gutiérrez Abascal 2, 28006 Madrid, Spain; rafaz@mncn.csic.es \\ 2 Department of Zoology, Swedish Museum of Natural History, Frescativägen 40, 11418 Stockholm, Sweden \\ 3 IBMM, Université de Montpellier CNRS, ENSCM, 34095 Montpellier, France; \\ sebastien.dutertre@umontpellier.fr \\ * Correspondence: saabalde@gmail.com
}

Citation: Abalde, S.; Dutertre, S.;

Zardoya, R. A Combined

Transcriptomics and Proteomics

Approach Reveals the Differences in the Predatory and Defensive Venoms of the Molluscivorous Cone Snail Cylinder ammiralis (Caenogastropoda: Conidae). Toxins 2021, 13, 642.

https://doi.org/10.3390/

toxins 13090642

Received: 10 August 2021

Accepted: 5 September 2021

Published: 10 September 2021

Publisher's Note: MDPI stays neutral with regard to jurisdictional claims in published maps and institutional affiliations.

Copyright: (c) 2021 by the authors. Licensee MDPI, Basel, Switzerland. This article is an open access article distributed under the terms and conditions of the Creative Commons Attribution (CC BY) license (https:/ / creativecommons.org/licenses/by/ $4.0 /)$.

\begin{abstract}
Venoms are complex mixtures of proteins that have evolved repeatedly in the animal kingdom. Cone snail venoms represent one of the best studied venom systems. In nature, this venom can be dynamically adjusted depending on its final purpose, whether to deter predators or hunt prey. Here, the transcriptome of the venom gland and the proteomes of the predation-evoked and defensive venoms of the molluscivorous cone snail Cylinder ammiralis were catalogued. A total of 242 venom-related transcripts were annotated. The conotoxin superfamilies presenting more different peptides were O1, O2, T, and M, which also showed high expression levels (except T). The three precursors of the J superfamily were also highly expressed. The predation-evoked and defensive venoms showed a markedly distinct profile. A total of 217 different peptides were identified, with half of them being unique to one venom. A total of 59 peptides ascribed to 23 different protein families were found to be exclusive to the predatory venom, including the cono-insulin, which was, for the first time, identified in an injected venom. A total of 43 peptides from 20 protein families were exclusive to the defensive venom. Finally, comparisons of the relative abundance (in terms of number of peptides) of the different conotoxin precursor superfamilies showed that most of them present similar abundance regardless of the diet.
\end{abstract}

Keywords: Conidae; transcriptome; proteome; conotoxin; cono-insulin; Cylinder ammiralis

Key Contribution: We analyze here the venom of the molluscivorous cone Cylinder ammiralis, using transcriptomics and proteomics. This is the first time the composition of the predatory and defensive venoms of a mollusk-hunter cone snail are analyzed in detail, and we highlight those proteins found exclusively in each of them.

\section{Introduction}

Venom systems are chemical weapons that confer a selective advantage and have evolved repeatedly in different animal lineages [1,2]. The best-characterized venom systems are arguably those of snakes [3] within vertebrates and those of cone snails [4] and spiders [5] within marine and terrestrial invertebrates, respectively. Animal venoms are complex mixtures dominated by peptides, and certain groups can dynamically adjust their venom's composition depending on external stimuli and whether they are used to capture prey or deter predators [6]. Venoms are a rich natural source for the discovery of drugs with painkiller, anti-inflammatory, or antihypertensive properties, and thus, they have great potential for human therapeutics [7]. Hence, there is active research devoted to catalogue animal venoms. 
The powerful combination of high-throughput proteomic and transcriptomic approaches has become the most efficient methodology to disentangle the highly complex mixtures of venoms and identify their components [8]. The advent of RNA sequencing [9] opened the door to identify the complete set of transcripts that are expressed in the venom gland of a venomous animal. These transcripts encode for toxins, as well as for proteins involved in the folding and maturation of the toxins [10]. Comparisons with other transcriptomes provide relevant information about the evolution and relative importance of these proteins. If comparisons involve other tissue transcriptomes, it is possible to uncover those genes that are essential for venom production, as well as variations in their expression in response to different stimuli [3]. If comparisons are between venom gland transcriptomes of related species, it is possible to discriminate those toxins that are shared-derived from common ancestors from those that are exclusive of a given species, as well as uncover instances of convergence [11]. In addition, a fraction of the venom gland transcripts is expressed in rather small quantities, whereas others may be even degraded before translation [12]. Moreover, the set of proteins that are synthesized in the venom gland do not necessarily match with the actual toxins found in the secreted mature venom, as the individual can dynamically adjust its composition depending on the stimuli [13]. Hence, it is important to also determine the venom gland proteome through mass spectrometry and compare it with the corresponding transcriptome to define the functional composition of the venom, including the identification of numerous post-translational modifications [14]. Finally, venom glands show regionalization of toxin production $[6,15]$, which can be best characterized combining transcriptomics and proteomics approaches [16].

The more than 900 described species of cone snails live in tropical and subtropical marine waters around the world [17]. They use venom in a very versatile way, as they have diverse hunting behaviors [18]; can prey on worms, snails, or fishes [19,20]; and can also use their venom to deter predators [6]. Each species synthesizes in a specialized venom gland a complex mixture of hundreds of peptides generally known as conotoxins that block neuromuscular receptors and ion channels to produce different physiological effects, including sedation, paralysis, or even death [21]. Conotoxins are first synthesized as precursors with signal, propeptide, and mature regions. The signal region is the most conserved and is used to classify the precursors into superfamilies [21]. After cleavage, the mature region becomes the functional toxin and is often characterized by specific disulfide bonds and post-translational modifications. In addition to conotoxins, species of the genus Gastridium, which engulf schools of small fishes with the rostrum before injecting venom through the proboscis [18], have evolved a specialized strategy to sedate fish prey that includes an insulin mimic to cause hypoglycemic shocks [22]. Interestingly, the monomeric structure of this insulin has been used to design a small and fully active insulin analog to treat human diabetes [23]. Apart from peptides, the venom of the West African species Genuanoconus genuanus produces a guanine derivative, genuanine, with toxic properties, indicating that the overall complexity of cone snail venom cocktails is far from being fully described [24].

Thus far, most studies on cone snail venomics either describe the transcriptome or the proteome of a given species. The full sets of transcripts encoding conotoxin precursors have been characterized in vermivorous [25], molluscivorous [26], and piscivorous [15,27] species, showing striking differences in composition. For instance, distinct members of the A superfamily are found in the venom of piscivorous Chelyconus ermineus [15] and Pionoconus magus [27]. Similarly, the toolkits of mature peptides of species with different diets are being catalogued from proteomes (e.g., Reference [28]). In the case of cono-insulin, this hormone has been annotated in the venom gland transcriptome from several species [22,29], and the presence of the actual protein in the venom gland was later confirmed [30]. However, this protein remains to be detected in the proteome of an injected venom, confirming its utilization during prey hunt. As in the case of snakes [31], the best results are achieved by combining both approaches, and thus, studies determining simultaneously both transcriptomes and proteomes of cone snails are becoming the standard [16,32-34]. 
Here, we performed a combined transcriptomic and proteomic approach to characterize the composition of the venom produced by the admiral cone snail, Cylinder ammiralis, a molluscivorous species of wide distribution in the Indo-Pacific region. For the proteome investigation, we milked predation-evoked and defense-evoked venoms and compared them with the venom gland transcriptome, revealing differences in composition. Moreover, we detected, for the first time, a cono-insulin in the predation-evoked venom of a cone species and demonstrated the use of hormone-like conopeptides during prey capture.

\section{Results}

\subsection{Composition of C. ammiralis Venom}

\subsubsection{Venom Gland Transcriptome}

The transcriptome of the venom duct from one specimen of Cylinder ammiralis was sequenced, generating 31.7 million reads. A total of 800,729 reads $(2.52 \%)$ were removed during the cleaning step, and the remaining reads were assembled into 86,230 contigs. BLASTX (National Center for Biotechnology Information (NCBI), Bethesda, MD, USA) searches against the custom database returned 1486 hits. After removing false positives, sequences with low coverage ( $<5$ mapped reads), assembly artifacts, truncated peptides ( $<45 \%$ of the length), and duplicated sequences, the transcripts of 242 conotoxin precursors, hormones, and venom-related proteins were annotated, with most of them being full-length $(72.72 \%)$. The majority of the incomplete peptides lacked the N-terminus, whereas only $17(7.02 \%)$ had no stop codon. All information related to these sequences is available in Supplementary Materials Table S1 and File S1.

A total of 209 conotoxin precursors were classified into 35 superfamilies based on the similarities of their signal regions. The most abundant superfamilies (those presenting more peptides) were $\mathrm{O} 1$ (35 conotoxin precursors), $\mathrm{O} 2$ (35), $\mathrm{T}(24)$, and $\mathrm{M}$ (16), which added up more than half of all identified conotoxin precursors (52.6\%; Figure 1A, expanded in Supplementary Materials Figure S1). All other superfamilies had less than ten precursors, and 24 of them were represented by less than five. Only one out of the five conotoxins (belonging to $\mathrm{A}$ and $\mathrm{O} 1$ superfamilies) previously reported for $\mathrm{C}$. ammiralis in ConoServer (http:/ / www.conoserver.org/, accessed on 7 August 2021) [35] was recovered in this study (Supplementary Materials File S2).

The relative expression level of each conotoxin precursor superfamily was calculated as the sum of the expressions of all its members. Three of the superfamilies with more peptides were also recovered among the most expressed (Figure 1B): O2 (39.49\% of the expression), O1 (28.77\%), and M (9.03\%). Despite its high diversity of peptides, the $\mathrm{T}$ superfamily only accounted for $1.95 \%$ of the overall conotoxin precursor expression, almost at the same level of the $\mathrm{H}$ superfamily $(1.92 \%$, seven conotoxin precursors). The three precursors of the J superfamily represented the third highest expression level $(9.57 \%)$. Cerm 08 had an intermediate expression level (3.24\%), and all other conotoxin precursor superfamilies showed expression levels lower than $1.5 \%$, with 18 of them below $0.05 \%$ (Figure 1B). A more detailed representation of these results can be found in Supplementary Materials Figure S2. The 20 most expressed superfamilies (accounting for $71 \%$ of overall conotoxin precursor expression) are shown in Supplementary Materials File S3.

The BLASTX searches identified 13 hormone-like conopeptides in the venom gland transcriptome of C. ammiralis (Supplementary Materials Table S1): two Conopressins, three Conorfamides, two Insulins, four Prohormone-4, and two Thyrostimulins (alpha and beta). In addition, 20 venom-related proteins were identified (Supplementary Materials Table S1), of which Ferritin (four paralogs) and Protein Disulfide Isomerase (PDI; nine paralogs) were the two most common. 
A

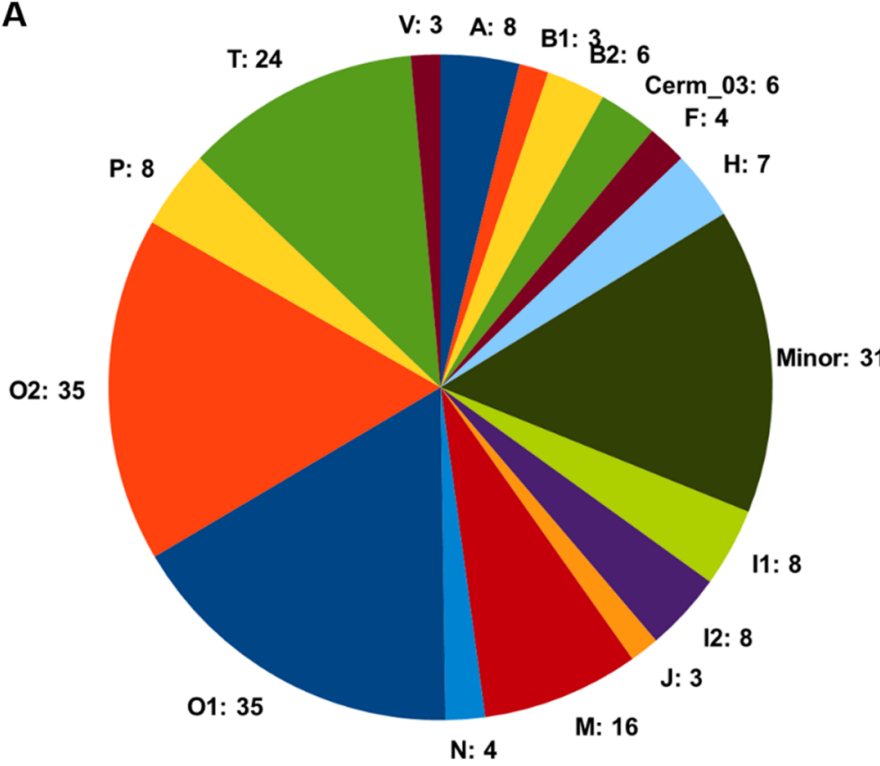

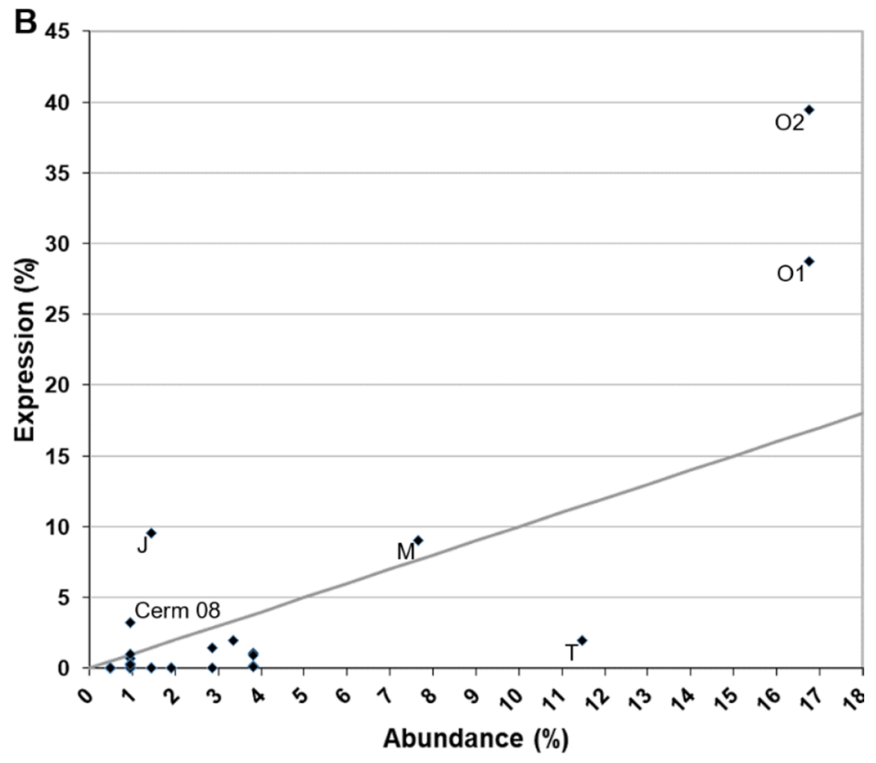

Figure 1. Summary of the diversity of conotoxins of Cylinder ammiralis based on the venom gland transcriptome. (A) Distribution in superfamilies of the 209 identified conotoxin precursors. The number beside the name represents the number of peptides within the superfamily. The minor superfamilies are expanded in Supplementary Materials Figure S1. (B) Scatter plot displaying the correlation between the number of peptides and the relative expression (measured in Transcripts Per Million, TPMs) for each superfamily. The two values were transformed to percentage. The gray line represents the 1:1 correlation between the two axes.

\subsubsection{Mass Spectrometry Analysis of Predatory and Defensive Venoms}

The predation- and defense-evoked venoms of $C$. ammiralis were collected separately. Both extracts showed markedly distinct yet equally complex profiles (Figure 2). A total of 217 different peptides were identified, of which only about half $(115$ or $53 \%)$ were common to the two venoms (Figure 3 and Supplementary Materials Table S2). Using the transcriptome as a reference, as well as blast searches against GenBank (https:/ / www.ncbi. nlm.nih.gov/genbank/, accessed on 7 August 2021) and UniProt (https:/ / www.uniprot. org/, accessed on 7 August 2021), all but 35 peptides could be annotated and assigned to 47 different (super)families. The ten most common protein families were $\mathrm{M}$ (nine peptides), O1 (nine), von Willebrand factor (eight), O2 (seven), T (seven), I1 (five), Angiotensin (four), Collagen alpha-4 (three), J (three), and PDI-A2 (three).

The predatory venom included 174 peptides, which were classified into 56 different protein families (except for 26 that remained unidentified; Figure 3 and Supplementary Materials Table S2). Conotoxins represented the main component of this venom, with 86 members. The (super)families with more peptides were O2 (20 peptides), O1 (14), M (13), T (ten), I1 (seven), and von Willebrand factor (eight). A total of 59 peptides ascribed to 23 different protein families were found to be exclusive to the predatory venom (Figure 3), including Actin, Coagulation factor, E superfamily, Elongation factor, I4 superfamily, Insulin (highlighted in Figure 2), and Synaptic vesicle membrane protein, with each one represented by one single peptide (Supplementary Materials Table S2).

Analysis of the defensive venom recovered 158 peptides, which were classified into 57 protein families (except for 23 that remained unidentified; Figure 3 and Supplementary Materials Table S2). The most common conotoxin superfamilies were O1 (14), O2 (12), $\mathrm{M}$ (ten), $\mathrm{T}$ (seven), and I1 (six). A total of 43 peptides from 20 protein families were exclusive to the defensive venom, including Calreticulin, Conodipine, FK506-binding protein, Galactose-binding, I2 superfamily, PLAT-LH2, Thioredoxin peroxidase, Tpra 06 superfamily, and U superfamily (Supplementary Materials Table S2). 

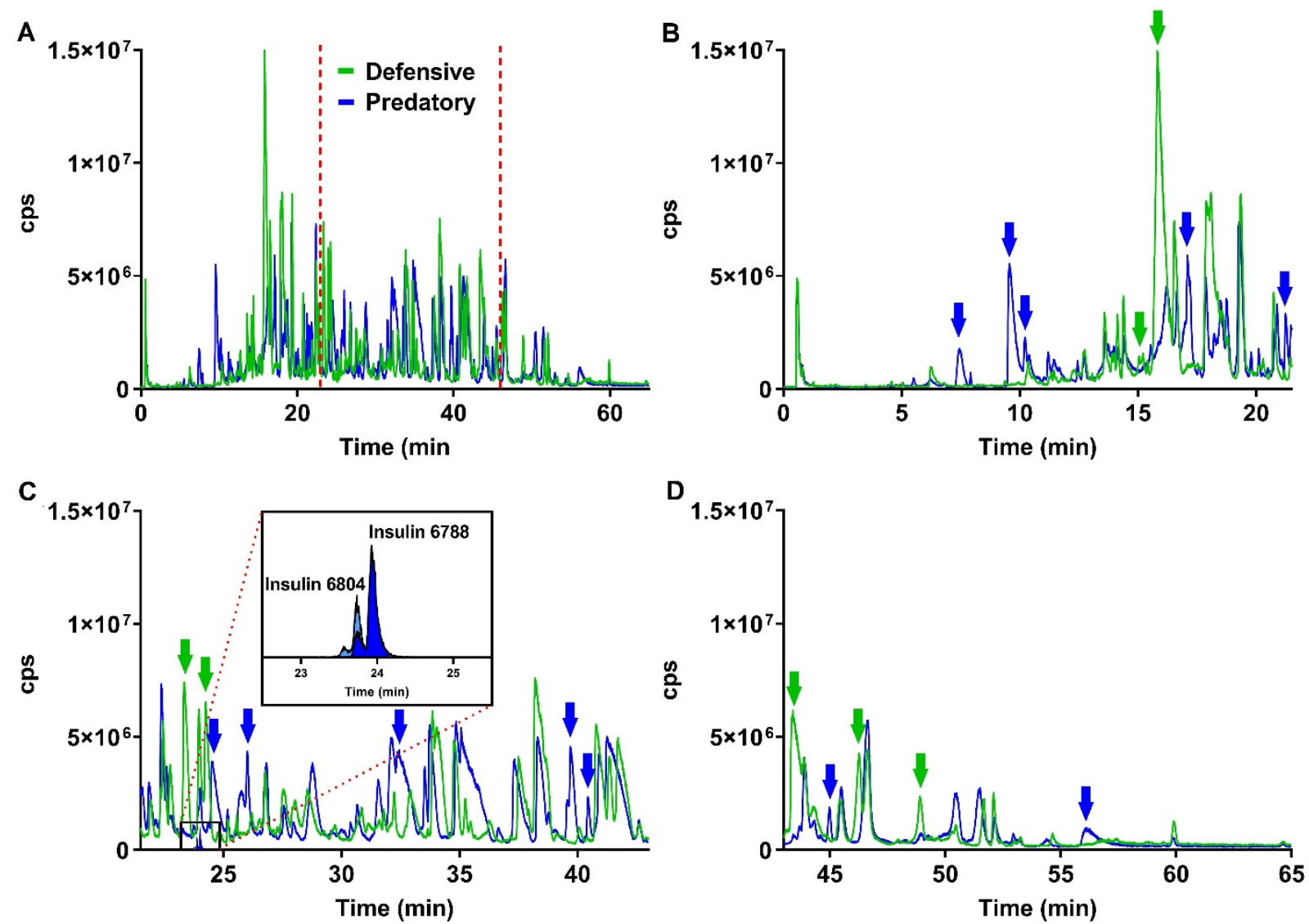

Figure 2. (A) LC-MS profiles of the predatory (blue) and defensive (green) venoms of Cylinder ammiralis. The red dashed lines mark the limits of the expanded sections in (B-D). The arrows highlight the main differences between the two venoms, using the same color code. In the panel $(\mathbf{C})$, the masses of the insulin are shown in the inset.

\subsubsection{Transcriptome versus Proteomes}

Of the 374 venom components annotated in this study, 85 were common to both the transcriptome and proteome approaches (Figure 3). These components included 19 conotoxin superfamilies, all the hormones, and four venom-related proteins (CAP, Condipine, Neuropeptide F, and PDI-A2; Supplementary Materials Table S2). Moreover, 132 out of the 217 peptides (61\%) were not annotated in the transcriptome by using the custom database (Figure 3; Supplementary Materials Table S2). Conversely, 157 out of 242 transcripts $(65 \%)$ were absent from the two proteomes (Figure 3; Supplementary Materials Table S2). The proteins annotated only in the proteome were classified into 36 different venom-related protein families. Most of the families found exclusively in the transcriptome had few members: B2 and Cerm 03 had six peptides each, and the other 12 superfamilies presented less than five peptides (Supplementary Materials Table S2). Six venom-related protein families were only found in the transcriptome, including two variants of the abundant PDI and the Ferritin (Supplementary Materials Table S2). 


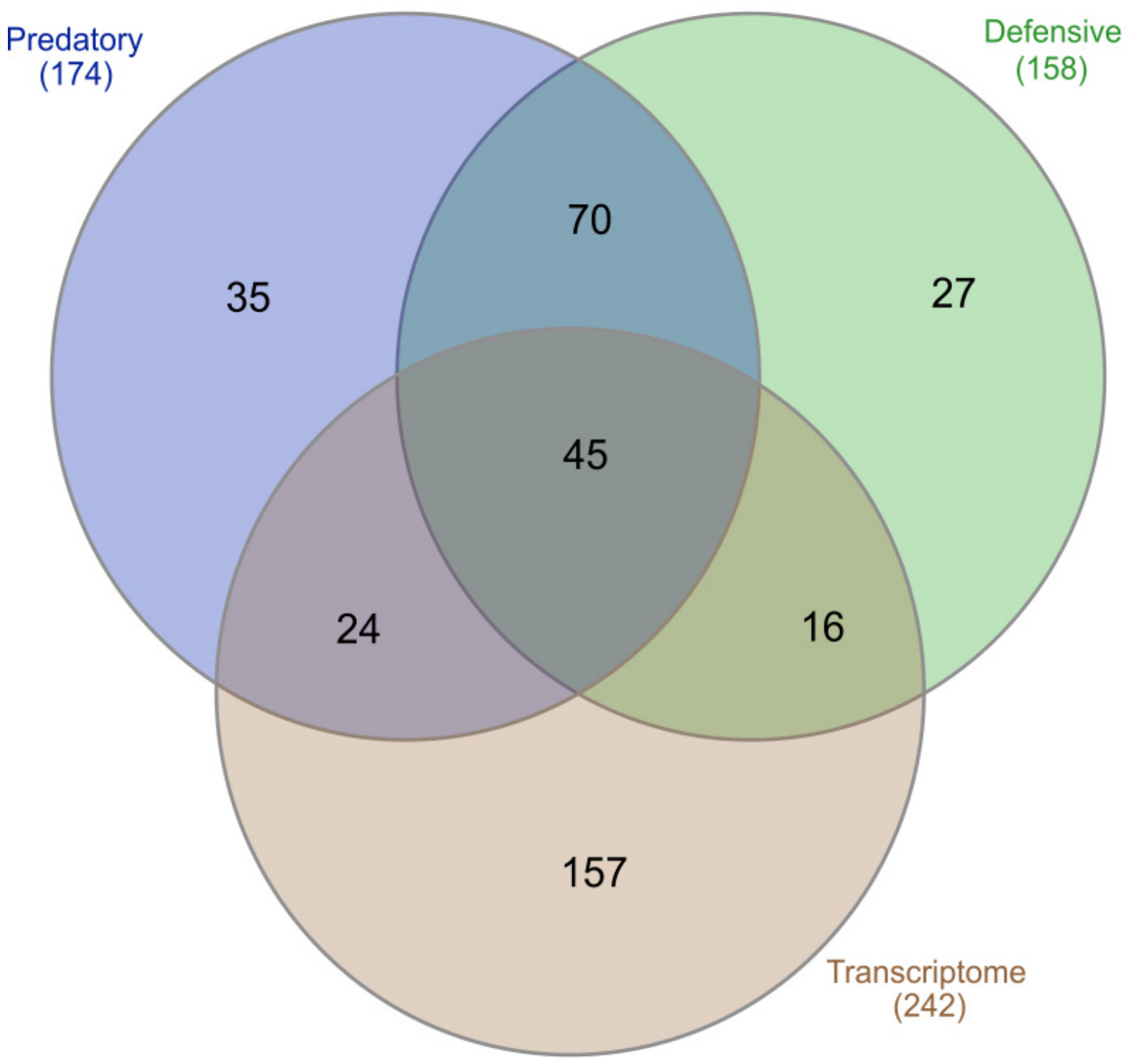

Figure 3. Number of unique and shared proteins found in the predatory venom (blue), defensive venom (green), and transcriptome (brown). The number under the category name represents the total number of proteins annotated for each dataset. The Venn diagram was built by using InteractiVenn (http:/ / www.interactivenn.net, accessed on 7 August 2021) [36].

\subsection{Differences in Venom Composition across Diets}

The compositions of the venom gland transcriptomes of $C$. ammiralis and other cone snails with different diets (six molluscivorous, six vermivorous, and six piscivorous) were compared, aiming at identifying conotoxin precursor superfamilies that appear significantly abundant (in terms of number of peptides) in a given diet. The comparison included 69 conotoxin precursor superfamilies, of which 55 showed no statistically significant differences between diets (Supplementary Materials Table S3). For six conotoxin precursor superfamilies, differences in abundance were significant only when the two most extreme cases were compared (i.e., the two diets with the highest and lowest number of peptides and excluding the third diet with intermediate abundance; Figure 4A and Supplementary Materials Table S3). The abundance of Cerm 01 and Con-ikot-ikot superfamilies was statistically significantly lower in molluscivorous than in piscivorous cones; the abundance of $C$ and I3 superfamilies was statistically significantly lower in molluscivorous than in vermivorous cones; finally, the I 2 and T superfamilies were significantly more abundant (in average 4.4 and 1.7 times, respectively) in molluscivorous than in piscivorous cones (Figure 4A and Supplementary Materials Table S3). 

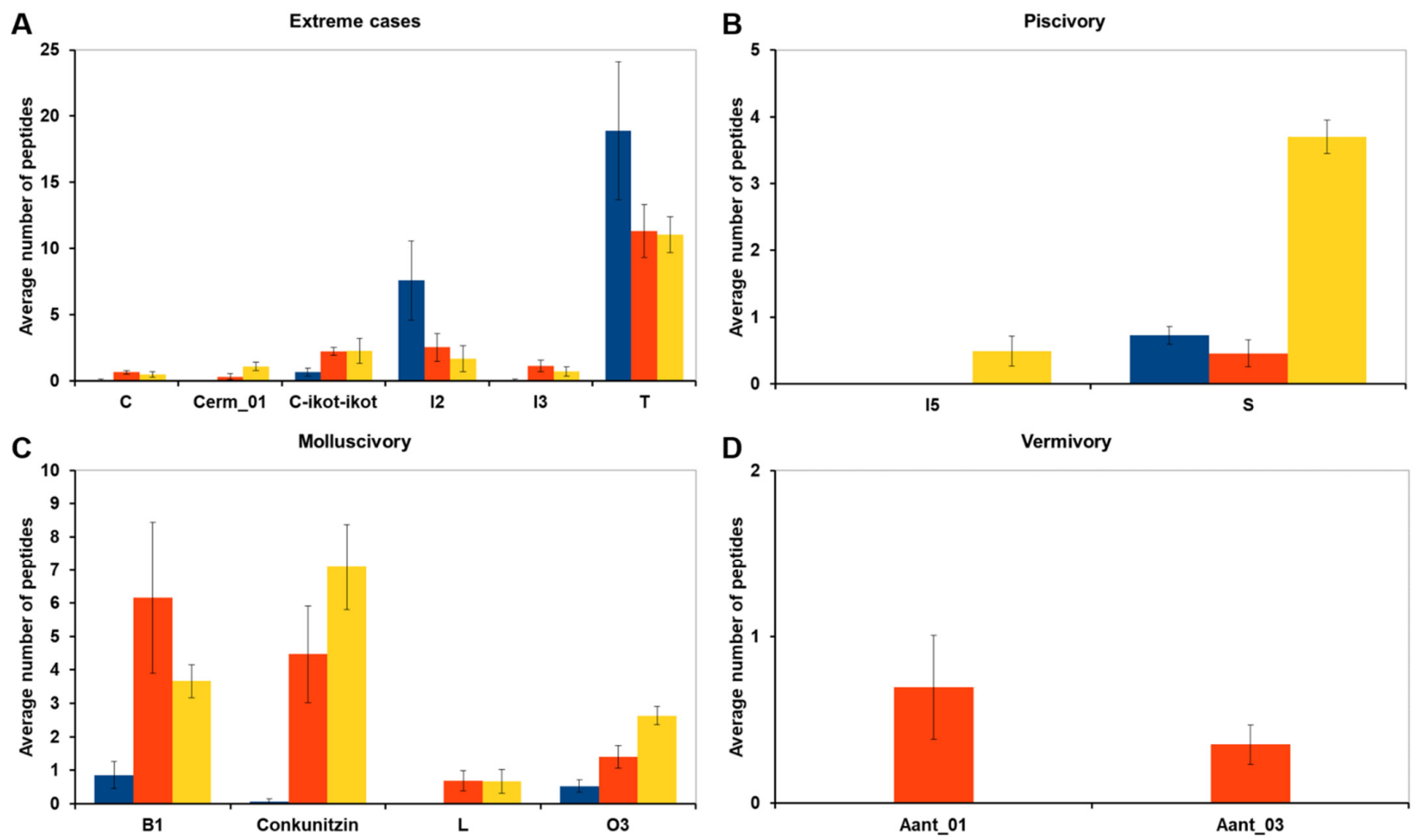

Figure 4. Summary of the superfamilies whose abundance is statistically different among diets. The three diets are shown in blue (molluscivory), orange (vermivory), and yellow (piscivory). The bars represent the average number of peptides per diet, and the error bars the standard error on the mean. (A) The differences were statistically different only between the lowest and the highest abundances. In the other three panels, the abundance of each superfamily was statistically different in one of the diets respect to the other two: (B) piscivory, (C) molluscivory, and (D) vermivory.

The abundance of eight conotoxin precursor superfamilies was statistically significantly different in one diet compared to the other two (Figure 4B-D). The I5 and S superfamilies showed a statistically significant higher abundance in piscivorous cones (Figure 4B). Although the differences in the I5 superfamily were subtle, as it was present only in individuals from Pionoconus magus, the differences in the abundance of the $\mathrm{S}$ superfamily were particularly relevant, presenting, on average, five times more conotoxins in piscivorous than in molluscivorous or vermivorous species (Figure 4B and Supplementary Materials Table S3). The Conkunitzin, $\mathrm{L}$, and $\mathrm{O} 3$ superfamilies had a statistically significant lower abundance in molluscivorous cone snails (Figure 4C and Supplementary Materials Table S3). Finally, Aant 01 and Aant 03 superfamilies (unassigned superfamilies in Reference [11], and here named after Africonus antoniomonteroi) were statistically more abundant in vermivorous species (Figure $4 \mathrm{D}$ and Supplementary Materials Table S3).

Overall, molluscivory was involved in all the above-described 14 significant abundance difference comparisons. This diet presented a significantly lower number of peptides than piscivorous and vermivorous cones in 12 out of the 14 superfamilies. The abundance of the $\mathrm{S}$ superfamily was much lower in molluscivorous than in piscivorous cones, and just slightly higher than in the vermivorous. The only exception to this trend were the I2 and T superfamilies with higher abundances in molluscivorous species; however, the standard deviation in these cases was between one-fourth and one-third of the average number of peptides, indicating a high variability among specimens (Figure 4A and Supplementary Materials Table S3). 


\section{Discussion}

\subsection{Transcriptomics and Proteomics, Two Complementary Approaches to Determine Venom Composition}

The venoms of each of the $>900$ species of cone snails are a rich natural source of potential medical drugs [7]. However, isolating and characterizing the functional constituents of cone snail venoms is challenging, as they are peptide cocktails of complex and dynamic composition. A combined transcriptomic and proteomic approach is currently the most efficient method to catalogue the peptides that are produced by an individual in the venom gland, as well as the fraction that is loaded into the harpoons to capture prey or deter predators $[6,16]$. Here, we show that results obtained by using transcriptomics and proteomics in identifying the venom toolkit of the admiral cone snail, C. ammiralis, are complementary. Nevertheless, there is a possibility that differences in transcriptomic and proteomic data are due to variability associated to the comparison of individuals from different locations. A total of 242 conotoxin precursors, hormones, and venomrelated proteins were identified in the transcriptome of the venom gland, a number that fits well within the range reported for other species of cone snails, such as P. magus [27], D. betulinus [25], and Varioconus guanche [11]. Similarly, a total of 217 different peptides were identified in the predatory and defensive proteomes of $C$. ammiralis, also within the expected range $[28,32]$.

Despite that the numbers recovered by the two approaches are very similar, only about a half of the venom components were common to both. As it has been shown in other studies, the high level of intra-specific variation makes the venoms from different individuals virtually different $[15,25,37]$. Hence, the differences observed between the two approaches could be attributed to the proteomes and the transcriptome coming from individuals from different localities. However, since the proteomic data were assembled and curated by using this same transcriptome as reference, all proteins annotated in the two proteomes should also be present in the transcriptome. This discrepancy might be explained by the intrinsic differences of the reference databases used to annotate the transcriptome and the proteomes. All the peptides identified in the proteome and not the transcriptome belong to venom-related proteins, with the exception of one peptide present in both the predatory and defensive venoms that belong to $\mathrm{R}$ superfamily, which was recently described in the vermivorous Conasprelloides villepinii and Gradiconus anabathrum, and it is hypothesized to block potassium channels [38]. Instead, the reference database used to annotate the transcriptome is mostly focused on conotoxin precursor superfamilies, several hormones, and a few known venom-related proteins, which indeed form most of the 85 proteins found in common to both approaches. The transcriptome and the proteomes shared most diverse superfamilies, such as $\mathrm{O} 1, \mathrm{O} 2, \mathrm{~T}$, and $\mathrm{M}$. These superfamilies tend to be the majority in all species, and they have been proposed to constitute the basic toolkit of the cone snail venom; moreover, they could be already present in the ancestor of the group [11]. In C. ammiralis, O2, O1, and M superfamilies were highly expressed, but not the T superfamily. In contrast, the J superfamily, with only three transcripts, showed the third highest expression level. The expression of these four superfamilies is remarkable, accumulating in only 20 peptides more than $70 \%$ of the total conotoxin precursor expression, and prompting a more careful study of these peptides. In particular, the J superfamily has been reported to be widespread in vermivorous cone snails, such as Strategoconus planorbis and Strategoconus ferrugineus [39]; Tesselliconus eburneus [40]; D. betulinus [25], Turriconus praecellens [37]; molluscivorous cone snails, such as C. victoriae [41]; and in piscivorous cone snails, such as C. ermineus [15] and P. magus [27]. The conotoxins of the J superfamily are one of the few that block both voltage-gated $\left(\mathrm{K}^{+}\right)$and ligand-gated (nicotinic acetylcholine) ion channels [39]. The high expression level of this superfamily, its presence in both proteomes, and its widespread distribution among diets might be an indicator of the importance of this superfamily to capture prey and deter predators in this species. 
The annotation of several proteins exclusively in the transcriptomic approach was not related to a particularly low expression of the corresponding transcripts, as many conform to average expression levels of the whole transcriptome, and, for instance, conotoxin precursor OK053_207, belonging to the O1 superfamily, was actually the transcript with the highest expression in the transcriptome $(4.68 \%)$. In this regard, it is important to note that transcriptome assembly and annotation are not straightforward steps, and some extra transcripts may correspond to assembly artifacts or background expression of housekeeping genes [42].

Based on BLAST searches against the complete NCBI and UniProt databases, we identified up to 6964 open reading frames (ORFs) in the transcriptome of C. ammiralis. Apart from conotoxin precursor superfamilies, hormones, and the few known venomrelated proteins, it is difficult to determine whether all other ORFs inferred in the venom gland transcriptome are venom-related or housekeeping proteins. In this regard, the list of proteins annotated in the proteome is key to identify those proteins that are functional in the secreted venom and help completing the annotation of the venom gland transcriptome. In fact, the annotation of the complete transcriptome included the identification of twothirds of the 36 protein families annotated only in the proteome, whereas 12 of them remained elusive, stressing the importance of combining the two approaches to obtain a complete picture of venom composition [42]. In addition, future comparisons to other tissue transcriptomes could help identifying those transcripts (proteins) that are exclusively or differentially overexpressed in the venom gland.

\subsection{Predatory- versus Defensive-Evoked Venoms}

A classic histological study of the venom gland of $P$. magus already showed that the proximal and distal regions could produce venoms with different pharmacological properties [43]. More recently, the regionalization of venom production along the venom gland was associated to predatory- (distal region) and defensive-evoked (proximal region) responses in Gastridium geographus [6]. Here, we studied the predatory- and defensiveevoked venoms of $C$. ammiralis. Both venoms shared 115 peptides, which represent $57 \%$ of the predatory venom and $75 \%$ of the defensive venom. The O1, O2, M, and T superfamilies were the most common in the shared fraction. Another peptide that was relatively diverse in both the predatory and defensive venoms was the von Willebrand factor (VWF). The presence of this peptide is intriguing, as it is known to interact with platelets to initiate hemostatic plug formation. In the venom of snakes, C-type lectin-like proteins and $\mathrm{Zn}^{2+}$ metalloproteinases bind VWF and interfere with platelet aggregation [44]. The presence of neprilysin, a $\mathrm{Zn}^{2+}$ metalloproteinase, was also detected in both the predatory and defensive venoms.

Another protein related to hematologic disorders, the multiple coagulation factor deficiency protein 2 (MCFD2), was found exclusively in the predatory venom, suggesting that this venom might have hemorrhagic properties. All members of the A superfamily were detected in the predatory venom, but one was also found in the defensive venom. The A superfamily, together with the S superfamily, has been recognized as particularly determinant for the toxic effect of the venom of piscivorous cone snails [15,27]. One member of the E and I4 superfamilies was isolated exclusively in the predatory venom. These conotoxins were also reported in two other molluscivorous species, C. victoriae [41] and C. gloriamaris [26], although their function remains unknown. Finally, an insulin analog was isolated exclusively in the predatory venom. Although the expression of a specialized insulin in the venom duct of piscivorous Gastridium species was reported [22], this is the first time that the protein is isolated in a secreted venom and associated specifically to a predatory behavior. Moreover, the use of insulin was associated to the particular hunting behavior of Gastridium species, which engulf fishes with the rostrum and then inject the venom [18]. However, the use of insulin in a molluscivorous cone snail indicates that causing hypoglycemic shocks is a more general preying strategy. 
The defensive venom had exclusively the conodipine, a type of phospholipase A2 (PLA2), which has been found in species of the piscivorous genera Chelyconus and Pionoconus, vermivorous species of the genus Kioconus, and the molluscivorous species C. victoriae (see Reference [45] and the references therein). This enzyme catalyzes the $\mathrm{Ca}^{2+}$-dependent hydrolysis of phospholipids, and as a component of snake venom, it has myotoxic effects and produces local pain and inflammation [46]. Another interesting enzyme exclusive of the defensive venom was the Thioredoxin peroxidase (TPX), which catalyzes the reduction of hydrogen peroxide, protecting against tissue damage, and was reported in the venom gland of honey bee workers that use venom for defense [47]. The first peptide belonging to the U superfamily was reported in Cylinder textile, and its injection in mice triggered jumping and convulsion [48]. It was later found in C. victoriae [41], C. gloriamaris [26], and Leptoconus amadis [49]. Here, we detected this superfamily only in the defensive venom of $C$. ammiralis. Its ubiquity in molluscivorous species and its presence exclusive to the defensive venom suggest the importance of this superfamily in defensive responses within this group. Nevertheless, members of this superfamily were also identified in the venom gland transcriptomes of the vermivorous Indo-Pacific Turriconus praecellens [37] and West African Africonus and Varioconus [11].

Although proteomic analysis of the venom gland is a common approach to the characterization of venom composition, the common practice is the dissection of the venom gland, which does not allow us to make inferences about the specific toxins used during prey hunting or defense against predators. Regarding mollusk hunter cones, there are two cases where the injected venom was studied. The injected venom of $C$. textile was analyzed, showing variation in venom composition in successive injections [50]. As in C. ammiralis, this venom that would constitute the predatory venom of this species was rich in $\mathrm{M}, \mathrm{O}$, and $\mathrm{T}$ conotoxin superfamilies. Furthermore, the predatory and defensive venoms of Conus marmoreus have also been described [6]. The defensive venom of $C$. marmoreus was more complex than the predatory one, contrasting with the pattern observed in C. ammiralis. Sadly, these two studies were mostly focused on the general profile of the venom, and little detail about their composition is provided.

The compositions of the defensive and predatory venoms of C. ammiralis differ considerably from those of the corresponding venoms in the piscivorous Gastridium [6]. In the latter, the defensive venom was mainly composed by members of the O1, M, and A superfamilies, whereas the predatory venom contained mainly contryphan and conopressin/conophysin. All of these superfamilies were common to both venoms in C. ammiralis, except the A superfamily, which was found almost exclusively in the predatory venom, contrary to the observation in Gastridium [6]. The predatory venom of Rhombiconus imperialis, a cone snail species that preys on fireworms (family Amphinomidae), was reported to contain mainly members of the $\mathrm{T}$ and $\mathrm{K}$ superfamilies [16]. These striking differences in the composition of predatory and defensive venoms are likely associated with the different diets, hunting behaviors, and types of predator encountered by these species, emphasizing the need to better understand the ecology of the stunning diversity of cone snails.

\subsection{Comparative Analyses of Venom Composition across Diets}

The initial characterization of venom gland transcriptomes from cone snails was based on the 454-pyrosequencing technology, which did not require assembly procedures to obtain the conotoxin precursor sequences (e.g., Reference [51]). However, this technology is now discontinued and superseded by Illumina-based RNA sequencing (Illumina, San Diego, CA, USA), which is more powerful and capable of detecting virtually all transcripts (even those with low expression) but requires an assembly step (e.g., References $[15,25,26])$. As a consequence, the comparison of 454-based and Illumina-based venom gland transcriptomes is not straightforward. Moreover, the rapid accumulation of newly described conotoxin precursor superfamilies implies that these might be present but not annotated in older transcriptomes. Finally, the names of the superfamilies are 
not always consistent across studies, and careful comparison of annotations is required. Fortunately, Illumina-based venom gland transcriptomes are rapidly accumulating, and annotations are becoming standardized, thus allowing comparative studies on the composition of venoms across species [11,19]. These studies are fundamental for understanding the adaptive value of this important functional trait and its relation with dietary specialization [19]. Here, we compared the relative abundance of the different conotoxin precursor superfamilies (in terms of number of members) in the venom gland transcriptomes of 19 cone specimens, taking into consideration their diet (worms, snails, or fishes). The relative abundance of most conotoxin precursor superfamilies (55 out of $69 ; 80 \%$ ) was similar in the different species, regardless of the diet. Through natural selection, this master formula has been fine-tuned and adapted to specific prey and predators by developing a toxin variant capable of triggering different physiological responses, as well as modulating the abundance and expression of specific conotoxin precursor superfamilies in the different cone snail species. For instance, the $S$ superfamily contains, on average, five times more conotoxins in piscivorous than in molluscivorous or vermivorous species. The $S$ superfamily conotoxins have been described in many cone snail species and are involved in the inhibition of ligand-gated serotonin and nicotinic acetylcholine receptors [21]. In the piscivorous C. ermineus, this superfamily showed significantly higher expression in the distal region [15]. Venoms of molluscivorous species were characterized by significantly lower number of peptides than those of piscivorous and vermivorous cones in 12 out of 14 conotoxin precursor superfamilies. Only I2 and T superfamilies showed higher abundances in molluscivorous species, although standard deviations indicated high variability among specimens. The conotoxins of the T superfamily can have cysteine frameworks V and X [21]. The latter is typical of molluscivorous species, including C. marmoreus [40], Conus araneosus (Genbank entry AKJ51806), C. victoriae [41], and C. ammiralis (this work), but also found in the piscivorous P. magus [27]. Finally, the only two conotoxin superfamilies highlighted in vermivorous cones in respect to the other two are Aant 01 and Aant 03. However, these superfamilies have recently been described in West African cones [11] and thus far have been reported only in these species. No function is known to these conotoxins.

A principal component analysis (PCA) was used to analyze general differences in venom composition, and it showed a defined pattern between the three diets (Supplementary Materials Figure S3). Molluscivorous cones had a remarkable compact pattern, with $C$. ammiralis being the most separate species in the PCA. Similarly, piscivorous cones were recovered together, whereas the vermivorous cones were highly dispersed. Molluscivorous cones have a monophyletic origin [33], and independent origins have been postulated for Atlantic and Indo-Pacific piscivorous cones (represented by the two species here analyzed), respectively [15]. However, vermivory has been inferred to be the ancestral condition in cones and is widespread throughout the Conidae Tree of Life. Therefore, our sampling may have many gaps among vermivorous lineages, thus artificially inflating the differences within this group.

\section{Conclusions}

In this study, we characterized the venom gland transcriptome of the molluscivorous species C. ammiralis, together with the proteomic analysis of both its predatory and defensive venoms. Although a core of common components was identified, our results also demonstrate some purpose-specific conotoxin and venom proteins that are uniquely found in either the predatory or defensive venom. More work-particularly on the functional effects of several classes of conotoxins-is required for a better understanding of the evolution and specialization of these animals. Overall, this study highlights the necessity to combine venom gland transcriptomics and predatory and defensive venom proteomics with more ecology-based observations in order to draw meaningful conclusions on the purpose of each conotoxin type injected. 


\section{Materials and Methods}

\subsection{Taxon Sampling}

For transcriptome analyses, one adult specimen of $C$. ammiralis was captured in Kyoda Bay (Okinawa island, Japan), in 2017, with corresponding permits. This individual was dissected in a resting stage to remove the venom duct, which was stored in $1 \mathrm{~mL}$ of RNAlater (Invitrogen, Life Technologies, San Diego, CA, USA) at $4{ }^{\circ} \mathrm{C}$ during the sampling campaign and at $-20^{\circ} \mathrm{C}$ for the long-term. For proteome analyses, a total of two adult specimens of C. ammiralis were captured from the Southern Great Barrier Reef (Queensland, Australia), under the corresponding permits, and kept alive at the University of Queensland aquarium facility, with the temperature set to 23 to $24^{\circ} \mathrm{C}$, in a 12:12 light-dark cycle.

The species here studied belongs to the genus Leptoconus according to the morphologybased classification of cone snails into genera [52], but it belongs to the subgenus Cylinder according to the molecular-based classification of cone snails into subgenera [53]. To resolve this controversy, we extracted and annotated by using BLAST searches for the 13 protein-coding and two ribosomal RNA (rRNA) mitochondrial genes from the assembled transcriptome (see below). These protein-coding and rRNA genes were aligned with orthologs from other cone snail mitogenomes (Supplementary Materials Table S4; [54]), using TranslatorX (http: / / translatorx.co.uk, accessed on 7 August 2021) [55] and MAFFT v.7 (EMBL-EBI, Hinxton, United Kingdom) [56], respectively. The individual alignments were combined into a concatenated dataset, and best-fit partitions and substitution models were inferred with PartitionFinder v.1 (https:/ / www.robertlanfear.com/partitionfinder/, accessed on 7 August 2021) [57]. A maximum likelihood phylogenetic tree was reconstructed by using RaxML (https:/ / cme.h-its.org/exelixis/web/software/raxml, accessed on 7 August 2021) v. 8.1.16 [58]. The reconstructed tree recovered the species here studied deeply nested within a clade including several species of the genus Cylinder (Supplementary Materials Figure S4), and therefore it was renamed as Cylinder ammiralis (following a generic classification), and this name is used throughout the study.

\subsection{RNA Extraction and Sequencing}

The venom gland was incubated in $300 \mu \mathrm{L}$ of TRIzol (ThermoFisher Scientific, Waltham, MA, USA) and grinded with ceramic beads in a Precellys Evolution tissue homogenizer (Bertin Instruments, Montigny-le-Bretonneux, France). The solution was mixed with $60 \mu \mathrm{L}$ of chloroform and centrifuged at $12,000 \times \mathrm{g}$ for $15 \mathrm{~min}$ at $4{ }^{\circ} \mathrm{C}$. The supernatant was recovered, mixed with one volume of isopropanol, and left overnight at $-80{ }^{\circ} \mathrm{C}$. Total RNA was purified by using the DirectZol miniprep kit (Zymo Research, Irvine, CA, USA), following manufacturer's instructions.

Dual-index cDNA libraries were constructed by using the TruSeq RNA library Pep v2 (Illumina, San Diego, CA, USA), following manufacturer's instructions. After the quality assessment of the libraries, these and other samples were pooled and split into several runs of paired-end sequencing $(2 \times 100 \mathrm{bp})$ in an Illumina HiSeq2500 (each pool divided into two flow cells), following standard procedures at Sistemas Genómicos (Valencia, Spain).

\subsection{Transcriptome Assembly and Transcript Annotation}

The raw reads were quality checked by using FastQC (https: / /www.bioinformatics. babraham.ac.uk/projects / fastqc/, accessed on 7 August 2021) [59] and assembled by using Trinity v.2.6.6 (https:/ / github.com/trinityrnaseq/trinityrnaseq/releases/tag/Trinity-v2.6 .6, accessed on 7 August 2021) [60] with default parameters (minimum contig length: 200; sequence identity threshold: 0.95 ), and the Trimmomatic option active (to remove Illumina adapters, trim low-quality regions and discard short sequences; SLIDINGWINDOW: 4; LEADING: 5; TRAILING: 5; MINLEN: 25). Identification of open-reading frames (ORFs) in the assembled transcriptome was performed with TransDecoder 2.0.1 (https:// github.com/TransDecoder/TransDecoder, accessed on 7 August 2021) [61] and annotation with Trinotate 3.1.1 (https:/ / github.com/Trinotate/Trinotate.github.io/wiki, accessed on 7 August 2021) [62]. 
The amino acid sequences of conotoxin precursors, hormones, and venom-related proteins were downloaded on 24 May 2020 from GenBank release 237, Uniprot release 2020_02, and ConoServer release 24 May 2020. The three databases were concatenated, and duplicates were removed. The resulting file was formatted as a BLAST+ database [63]. The transcripts encoding putative conotoxin precursors, hormones, and venom-related proteins of $C$. ammiralis were identified through BLASTX similarity searches of the assembled contigs against this database (e-value: $1 \times 10^{-5}$ ). All contigs that were hit were manually inspected to remove false positives and to extract the correct CDS regions, creating a working list of proteins. Afterwards, several filtering steps were implemented. Assembly artifacts, such as chimaeras and sequences with low-coverage ( $<5$ reads mapped), were detected in Tablet 1.19.09.03 [64] after mapping raw reads against all selected sequences, using Bowtie2 (http: / / bowtie-bio.sourceforge.net/bowtie2/index.shtml, accessed on 7 August 2021) [65], and then discarded. Sequences with low coverage in terminal positions were trimmed to avoid the inclusion of spurious variability in conotoxin isoforms. Duplicated and highly truncated ( $>55 \%$ ) sequences were removed. The signal, propeptide, and mature regions of the remaining conotoxin precursors were identified by using the Conoprec tool (http: / / www.conoserver.org / ?page=conoprec, accessed on 7 August 2021) [35]. Conotoxin precursors were assigned to the corresponding superfamilies based on the identity of the signal region, using an approximate threshold of $75 \%$ divergence [25]. Approximate relative expression levels of the transcripts from the different superfamilies were estimated by mapping clean reads back to assembled contigs. TPM (transcripts per kilobase million), which normalize for gene length and sequencing depth, were estimated with the RNA-Seq by Expectation Maximization (RSEM) package included in Trinity v.2.6.6 [60].

\subsection{Venom Milking}

A live gastropod snail (Nassariidae or Strombidae) was used to lure the cone snails in the aquarium and elicit the predatory behavior. Milking was conducted by using a microcentrifuge tube covered with parafilm and a piece of the snail to initiate stinging and collect the predation-evoked venom. To force a defense behavior, the cone snails were removed from the tank and disturbed by applying light pressure to the shell with long forceps. Once the proboscis was extended and stinging started, the defense-evoked venom was collected by using a microcentrifuge tube covered with parafilm. Several stings from the same individual were pooled for each of the venoms. The collected venoms were centrifuged, lyophilized, and stored at $-20^{\circ} \mathrm{C}$.

\subsection{LC-MS and Proteomic Analysis}

The predation- and defense-evoked venom samples were subjected to liquid chromatography coupled with electrospray mass spectrometry (LC-ESI-MS) as previously described [6,16]. Briefly, 500 $\mu \mathrm{g}$ of each venom was loaded onto a Kinetex $\mathrm{C}_{18} 100 \AA$ column $(2.1 \mathrm{~mm} \times 150 \mathrm{~mm}, 3 \mu \mathrm{m})$ (Phenomenex, Torrance, CA, USA) fitted with a precolumn. The RP-UPLC runs were operated on an Acquity H-Class ultrahigh-performance liquid chromatography (UPLC) system (Waters, Corp., Milford, MA, USA) fitted with a UV detector (diode array detector) under the control of Waters MassLynx software version 4.1 (Waters, Corp., Milford, MA, USA). The samples were introduced into the mass spectrometer at a flow rate of $500 \mu \mathrm{L} / \mathrm{min}$ after their elution from the UPLC (gradient of $0-80 \%$ B $0.1 \%$ formic acid in acetonitrile in $80 \mathrm{~min}$ ). Acquisitions were carried out over the range $50 \mathrm{Da}$ to $1800 \mathrm{Da} m / z$ every $0.1 \mathrm{~s}$ on a Synapt-G2-S high-definition MS system (Waters, Corp., Milford, MA, United States). Molecular masses were obtained by analyzing each peak from the total ion current (TIC) chromatogram with Waters Mass Lynx software (version 4.1) (Waters, Corp., Milford, MA, USA).

Next, the venom peptide/protein extracts were denatured, reduced, alkylated, and subjected to shotgun proteomics, as already described [66]. Briefly, each sample $(\sim 50 \mu \mathrm{g})$ was dissolved in $89 \mu \mathrm{L}$ of triethylammonium bicarbonate (TEABC) $100 \mathrm{mM}$ and reduced with dithiothreitol (DTT) $1 \mathrm{M}$ for $30 \mathrm{~min}$ at $60{ }^{\circ} \mathrm{C}$. Alkylation was performed with iodoac- 
etamide (IAA) $0.5 \mathrm{M}$ (incubation for $30 \mathrm{~min}$ in the dark). Samples were enzymatically digested by adding $2 \mu \mathrm{g}$ of trypsin (Gold, Promega, Madison, WI, USA) in TEABC $100 \mathrm{mM}$ and incubating overnight at $30{ }^{\circ} \mathrm{C}$. After purification and concentration of the samples (OMIX Tips $\mathrm{C}_{18}$ reverse-phase resin, Agilent Technologies Inc., Santa Clara, CA, USA), peptides were dehydrated in a vacuum centrifuge and subjected to nano-flow liquid chromatography coupled to tandem mass spectrometry (NanoLC-MS/MS). Samples were resuspended in $20 \mu \mathrm{L}$ formic acid $(0.1 \%$, buffer $\mathrm{A})$, and $1 \mu \mathrm{L}$ was loaded onto an analytical $25 \mathrm{~cm}$ reversed-phase column (75 mm inner diameter, Acclaim Pepmap $100^{\circledR} \mathrm{C}_{18}$, Thermo Fisher Scientific). Samples then were separated with an Ultimate 3000 RSLC system (Thermo Fisher Scientific, Waltham, MA, USA) coupled to a Q Exactive HF-X (Thermo Fisher Scientific, Waltham, MA, USA) via a nano-electrospray source, using a 123 min gradient of $6 \%$ to $40 \%$ of buffer B ( $80 \%$ ACN, $0.1 \%$ formic acid) and a flow rate of $300 \mathrm{~nL} / \mathrm{min}$. For the MS/MS analyses, full scans $(375-1500 \mathrm{~m} / \mathrm{z})$ were acquired in the mass analyzer (Thermo Fisher Scientific, Waltham, MA, USA) with a 60,000 resolution at $200 \mathrm{~m} / z ; 3 \times 106$ ions were accumulated within a maximum injection time of $60 \mathrm{~ms}$ and detected in the mass analyzer. The twelve most intense ions with charge states $\geq 2$ were sequentially isolated to a target value of $1 \times 105$ with a maximum injection time of $45 \mathrm{~ms}$ and fragmented by higher-energy collisional dissociation (HCD) in the collision cell (normalized collision energy of 28\%) and detected in the mass analyzer at 30,000 resolution.

\subsection{Bioinformatic Integration of Proteomic and Transcriptomic Data}

All the contigs assembled in the transcriptome analyses were translated into the six reading frames, creating the database used during the elucidation of the MS spectra. MS/MS spectra and transcriptomic sequences were matched by using the PEAKS Studio 8.5 software (Bioinformatics solutions, Waterloo, ON, Canada) with carbamidomethylation as fixed modification, while oxidation (M) was set as variable modifications, with maximum missed cleavages set at 3 for trypsin digestion. Parent mass was set to $5 \mathrm{ppm}$, while fragment mass error tolerance was $0.015 \mathrm{Da}$. Inaccurate proteins were filtered out by using a false discovery rate (FDR) of $1 \%$, and there were two or more unique peptides. A $-10 \lg P$ $>120$ was used to estimate whether the detected proteins were identified by enough reliable peptides MS/MS spectra. Identification of additional mutations and sequence correction were performed with the Spider algorithm from PEAKS Studio software. This algorithm corrects the sequences stored in transcriptomic database with de novo sequences based on MS/MS spectra, which allowed us to identify post-translational modifications (PTMs) and mutations. Minimum ion intensity for mutation and PTMs was set to 5\%, and ALC score $\geq 90$ for de novo sequences, leading to low precursor mass error, in order to identify reliable PTMs and potential mutations.

\subsection{Analysis of Venom Composition across Diets}

The number of isoforms per conotoxin precursor superfamily was compared across cone snail species with different diets, using Illumina-based transcriptomic data. The following species represented molluscivorous cone snails: Cylinder ammiralis (this study), Cylinder gloriamaris [26], two Cylinder victoriae ([41] and ConoServer data i.e., the list of conotoxin precursors and conotoxins registered on ConoServer for this species), two Darioconus episcopatus ([67] and Conoserver data), and Conus marmoreus ([68] and ConoServer data). Vermivorous cone snail species were represented by Africonus maioensis, Kalloconus trochulus, and Varioconus mercator [11], Dendroconus betulinus [25], Turriconus praecellens [37], and Calamiconus quercinus [69]. Finally, three specimens of Chelyconus ermineus [15] and three of Pionoconus magus [27] were included to represent piscivorous cone snails (note that most previous transcriptomes reported for piscivorous species were based on 454 technology).

Once a list of contoxin precursor superfamilies was extracted for each specimen (Supplementary Materials Table S5), we tested for differences among diets by using the species as replicates in R [70] with the CRAN packages normtest [71] and dunn.test [72]. The normality of the data was checked by using the Shapiro-Wilk test, and once confirmed, 
the homocedasticity of the data was checked by using the Bartlett's test, which is suitable for comparisons between more than two conditions with different sampling sizes. To identify those superfamilies whose abundances were significantly different among diets, we ran an ANOVA test for those samples that had passed both tests, or a Kruskal-Wallis test if one of them had failed. The post hoc analyses selected to assess the pairwise differences among diets were the Bonferroni correction (ANOVA) and the Dunn's test (KruskalWallis). Finally, the superfamilies from this dataset with variance zero were removed, and a principal component analysis (PCA) was run by using the prcomp function in R (R Core Team, Vienna, Austria) and plotted with ggplot2 (https:/ /ggplot2.tidyverse.org, accessed on 7 August 2021) [73].

Supplementary Materials: The following are available online at https:/ / www.mdpi.com/article/10 .3390 /toxins13090642/s1. Supplementary Materials Figure S1: Extension of Figure 1, including a second panel with all superfamilies grouped within the "Minor" category. Supplementary Materials Figure S2: Extension of Figure 1, including a second panel with details of all superfamilies showing a correlation between the number of peptides and the relative expression (measured in TPMs) between zero and four. Supplementary Materials Figure S3: PCA analysis of diet composition, measured as the number of conotoxin precursors identified for each superfamily. Supplementary Materials Figure S4: Phylogenetic ML tree based on complete mitochondrial genomes (concatenated 13 protein-coding genes plus two ribosomal RNA), bootstrap values are indicated above each node and scale bar indicates substitutions/site. The silhouette indicating the molluscivorous clade was downloaded from PhyloPic (T. Cunha; http://phylopic.org/, accessed on 7 August 2021). The pictures of the shells are from Alexander Medvedev (www.coneshells-am.ru, accessed on 7 August 2021). Supplementary Materials Table S1: List of conotoxin precursors, hormones, and venom-related proteins found in the venom gland transcriptome of Cylinder ammiralis. Supplementary Materials Table S2: List of the protein families found in the predation-evoked and defensive-evoked venoms, as well as in the venom gland transcriptome. Supplementary Materials Table S3: Summary of the statistical analyses comparing the abundance of each conotoxin precursor superfamily across diets. Supplementary Materials Table S4: Mitochondrial genomes used in the phylogenetic analyses. Supplementary Materials Table S5: Venom composition of all specimens used in the comparative analysis. Supplementary Materials File S1: Alignments of conotoxin precursors, hormones, and associated venom proteins of Cylinder ammiralis with homologues from other cone snail species. Supplementary Materials File S2: Alignments of the five conotoxin precursors of Cylinder ammiralis present in ConoServer with the closest sequences found in the transcriptome. Asterisks represent different amino acids in a given position. Supplementary Materials File S3: Fasta file containing the top 20 most expressed transcripts in the venom gland of $C$. ammiralis.

Author Contributions: All three authors conceived the study, collected the specimens, analyzed the data, wrote down and approved the manuscript. S.A. obtained the transcriptomes and proteomes with support of all other authors. All authors have read and agreed to the published version of the manuscript.

Funding: This work was funded by the Spanish Ministry of Science and Innovation (CGL201675255-C2-1-P [AEI/FEDER, UE] and PID2019-103947GB-C22/AEI/10.13039/501100011033 to R.Z.; BES-2014-069575 and EST2019-013092-I to S.A.).

Institutional Review Board Statement: Not applicable.

Informed Consent Statement: Not applicable.

Data Availability Statement: The specimen of C. ammiralis analyzed in this study can be found in the Malacology collection of the National Museum of Natural Sciences (Madrid, Spain), under voucher number 15.05/87493. Raw reads were deposited at the SRA database with accession number SRR14921183, Bioproject PRJNA741614. Proteins identified in proteomic analyses were submitted to the NCBI database (accession numbers MZ484112-MZ484328). All protein sequences and their annotation can be assessed in the Supplementary Materials Tables S3 and S4.

Conflicts of Interest: The authors declare no conflict of interest. 


\section{References}

1. Modica, M.V.; Sunagar, K.; Holford, M.; Dutertre, S. Diversity and Evolution of Animal Venoms: Neglected Targets, Ecological Interactions, Future Perspectives. Front. Ecol. Evol. 2020, 8, 65. [CrossRef]

2. Holford, M.; Daly, M.; King, G.F.; Norton, R.S. Venoms to the rescue. Science 2018, 361, 842-844. [CrossRef] [PubMed]

3. Suryamohan, K.; Krishnankutty, S.P.; Guillory, J.; Jevit, M.; Schröder, M.S.; Wu, M.; Kuriakose, B.; Mathew, O.K.; Perumal, R.C.; Koludarov, I.; et al. The Indian cobra reference genome and transcriptome enables comprehensive identification of venom toxins. Nat. Genet. 2020, 52, 106-117. [CrossRef]

4. Pardos-Blas, J.R.; Irisarri, I.; Abalde, S.; Afonso, C.M.L.; Tenorio, M.J.; Zardoya, R. The genome of the venomous snail Lautoconus ventricosus sheds light on the origin of conotoxin diversity. Gigascience 2021, 10, giab037. [CrossRef] [PubMed]

5. Sanggaard, K.W.; Bechsgaard, J.S.; Fang, X.; Duan, J.; Dyrlund, T.F.; Gupta, V.; Jiang, X.; Cheng, L.; Fan, D.; Feng, Y. Spider genomes provide insight into composition and evolution of venom and silk. Nat. Commun. 2014, 5, 1-12.

6. Dutertre, S.; Jin, A.-H.; Vetter, I.; Hamilton, B.; Sunagar, K.; Lavergne, V.; Dutertre, V.; Fry, B.G.; Antunes, A.; Venter, D.J. Evolution of separate predation-and defence-evoked venoms in carnivorous cone snails. Nat. Commun. 2014, 5, 1-9. [CrossRef]

7. King, G. Venoms to Drugs: Venom as a Source for the Development of Human Therapeutics; Royal Society of Chemistry: London, UK, 2015; ISBN 1849736634.

8. Wilson, D.; Daly, N.L. Venomics: A mini-review. High-Throughput 2018, 7, 19. [CrossRef]

9. Nagalakshmi, U.; Waern, K.; Snyder, M. RNA-Seq: A method for comprehensive transcriptome analysis. Curr. Protoc. Mol. Biol. 2010, 89, 4-11. [CrossRef]

10. Barua, A.; Mikheyev, A.S. An ancient, conserved gene regulatory network led to the rise of oral venom systems. Proc. Natl. Acad. Sci. USA 2021, 118, e2021311118. [CrossRef]

11. Abalde, S.; Tenorio, M.J.; Afonso, C.M.L.; Zardoya, R. Comparative transcriptomics of the venoms of continental and insular radiations of West African cones. Proc. R. Soc. B 2020, 287, 20200794. [CrossRef]

12. Shyu, A.B.; Wilkinson, M.F.; Van Hoof, A. Messenger RNA regulation: To translate or to degrade. EMBO J. 2008, $27,471-481$. [CrossRef]

13. Yin, X.; Guo, S.; Gao, J.; Luo, L.; Liao, X.; Li, M.; Su, H.; Huang, Z.; Xu, J.; Pei, J. Kinetic analysis of effects of temperature and time on the regulation of venom expression in Bungarus multicinctus. Sci. Rep. 2020, 10, 1-11.

14. Hofmann, E.P.; Rautsaw, R.M.; Strickland, J.L.; Holding, M.L.; Hogan, M.P.; Mason, A.J.; Rokyta, D.R.; Parkinson, C.L. Comparative venom-gland transcriptomics and venom proteomics of four Sidewinder Rattlesnake (Crotalus cerastes) lineages reveal little differential expression despite individual variation. Sci. Rep. 2018, 8, 1-15.

15. Abalde, S.; Tenorio, M.J.; Afonso, C.M.L.; Zardoya, R. Conotoxin Diversity in Chelyconus ermineus (Born, 1778) and the Convergent Origin of Piscivory in the Atlantic and Indo-Pacific Cones. Genome Biol. Evol. 2018, 10, 2643-2662. [CrossRef]

16. Jin, A.-H.; Dutertre, S.; Dutt, M.; Lavergne, V.; Jones, A.; Lewis, R.J.; Alewood, P.F. Transcriptomic-proteomic correlation in the predation-evoked venom of the cone snail, Conus imperialis. Mar. Drugs 2019, 17, 177. [CrossRef]

17. Monnier, E.; Limpalaër, L.; Robin, A.; Roux, C. A Taxonomic Iconography of Living Conidae; ConchBooks: Harxheim, Germany, 2018; ISBN 9783939767923.

18. Olivera, B.M.; Seger, J.; Horvath, M.P.; Fedosov, A.E. Prey-capture strategies of fish-hunting cone snails: Behavior, neurobiology and evolution. Brain. Behav. Evol. 2015, 86, 58-74. [CrossRef] [PubMed]

19. Phuong, M.A.; Mahardika, G.N.; Alfaro, M.E. Dietary breadth is positively correlated with venom complexity in cone snails. BMC Genom. 2016, 17, 401. [CrossRef] [PubMed]

20. Duda, T.F.; Kohn, A.J.; Palumbi, R.S. Origins of diverse feeding ecologies within Conus, a genus of venomous marine gastropods. Biol. J. Linn. Soc. 2001, 73, 391-409. [CrossRef]

21. Robinson, S.D.; Norton, R.S. Conotoxin gene superfamilies. Mar. Drugs 2014, 12, 6058-6101. [CrossRef] [PubMed]

22. Safavi-Hemami, H.; Gajewiak, J.; Karanth, S.; Robinson, S.D.; Ueberheide, B.; Douglass, A.D.; Schlegel, A.; Imperial, J.S.; Watkins, M.; Bandyopadhyay, P.K. Specialized insulin is used for chemical warfare by fish-hunting cone snails. Proc. Natl. Acad. Sci. USA 2015, 112, 1743-1748. [CrossRef] [PubMed]

23. Xiong, X.; Menting, J.G.; Disotuar, M.M.; Smith, N.A.; Delaine, C.A.; Ghabash, G.; Agrawal, R.; Wang, X.; He, X.; Fisher, S.J.; et al. A structurally minimized yet fully active insulin based on cone-snail venom insulin principles. Nat. Struct. Mol. Biol. 2020, 27, 615-624. [CrossRef]

24. Neves, J.L.B.; Lin, Z.; Imperial, J.S.; Antunes, A.; Vasconcelos, V.; Olivera, B.M.; Schmidt, E.W. Small molecules in the cone snail arsenal. Org. Lett. 2015, 17, 4933-4935. [CrossRef] [PubMed]

25. Peng, C.; Yao, G.; Gao, B.-M.; Fan, C.-X.; Bian, C.; Wang, J.; Cao, Y.; Wen, B.; Zhu, Y.; Ruan, Z.; et al. High-throughput identification of novel conotoxins from the Chinese tubular cone snail (Conus betulinus) by multitranscriptome sequencing. Gigascience 2016, 5, 17. [CrossRef] [PubMed]

26. Robinson, S.D.; Li, Q.; Lu, A.; Bandyopadhyay, P.K.; Yandell, M.; Olivera, B.M.; Safavi-Hemami, H. The venom repertoire of Conus gloriamaris (Chemnitz, 1777), the glory of the sea. Mar. Drugs 2017, 15, 145. [CrossRef] [PubMed]

27. Pardos-Blas, J.R.; Irisarri, I.; Abalde, S.; Tenorio, M.J.; Zardoya, R. Conotoxin diversity in the venom gland transcriptome of the magician's cone, Pionoconus magus. Mar. Drugs 2019, 17, 553. 
28. Safavi-Hemami, H.; Siero, W.A.; Gorasia, D.G.; Young, N.D.; MacMillan, D.; Williamson, N.A.; Purcell, A.W. Specialisation of the venom gland proteome in predatory cone snails reveals functional diversification of the conotoxin biosynthetic pathway. $J$. Proteome Res. 2011, 10, 3904-3919. [CrossRef] [PubMed]

29. Safavi-Hemami, H.; Lu, A.; Li, Q.; Fedosov, A.E.; Biggs, J.; Showers Corneli, P.; Seger, J.; Yandell, M.; Olivera, B.M. Venom Insulins of Cone Snails Diversify Rapidly and Track Prey Taxa. Mol. Biol. Evol. 2016, 33, 2924-2934. [CrossRef]

30. Robinson, S.D.; Li, Q.; Bandyopadhyay, P.K.; Gajewiak, J.; Yandell, M.; Papenfuss, A.T.; Purcell, A.W.; Norton, R.S.; SafaviHemami, H. Hormone-like peptides in the venoms of marine cone snails. Gen. Comp. Endocrinol. 2017, 244, 11-18. [CrossRef]

31. Leonardi, A.; Sajevic, T.; Pungerčar, J.; Križaj, I. Comprehensive study of the proteome and transcriptome of the venom of the most venomous european viper: Discovery of a new subclass of ancestral snake venom metalloproteinase precursor-derived proteins. J. Proteome Res. 2019, 18, 2287-2309. [CrossRef]

32. Safavi-Hemami, H.; Hu, H.; Gorasia, D.G.; Bandyopadhyay, P.K.; Veith, P.D.; Young, N.D.; Reynolds, E.C.; Yandell, M.; Olivera, B.M.; Purcell, A.W. Combined proteomic and transcriptomic interrogation of the venom gland of Conus geographus uncovers novel components and functional compartmentalization. Mol. Cell. Proteom. 2014, 13, 938-953. [CrossRef]

33. Violette, A.; Biass, D.; Dutertre, S.; Koua, D.; Piquemal, D.; Pierrat, F.; Stöcklin, R.; Favreau, P. Large-scale discovery of conopeptides and conoproteins in the injectable venom of a fish-hunting cone snail using a combined proteomic and transcriptomic approach. J. Proteom. 2012, 75, 5215-5225. [CrossRef] [PubMed]

34. Jin, A.; Vetter, I.; Himaya, S.W.A.; Alewood, P.F.; Lewis, R.J.; Dutertre, S. Transcriptome and proteome of Conus planorbis identify the nicotinic receptors as primary target for the defensive venom. Proteomics 2015, 15, 4030-4040. [CrossRef] [PubMed]

35. Kaas, Q.; Yu, R.; Jin, A.H.; Dutertre, S.; Craik, D.J. ConoServer: Updated content, knowledge, and discovery tools in the conopeptide database. Nucleic Acids Res. 2012, 40, D325-D330. [CrossRef]

36. Heberle, H.; Meirelles, G.V.; da Silva, F.R.; Telles, G.P.; Minghim, R. InteractiVenn: A web-based tool for the analysis of sets through Venn diagrams. BMC Bioinform. 2015, 16, 1-7. [CrossRef] [PubMed]

37. Li, Q.; Barghi, N.; Lu, A.; Fedosov, E.A.; Bandyopadhyay, P.K.; Lluisma, O.A.; Concepcion, G.P.; Yandell, M.; Olivera, B.M.; Safavi-Hemami, H. Divergence of the Venom Exogene Repertoire in Two Sister Species of Turriconus. Genome Biol. Evol. 2017, 9 , 2211-2225. [CrossRef]

38. Möller, C.; Dovell, S.; Melaun, C.; Marí, F. Definition of the R-superfamily of conotoxins: Structural convergence of helix-loop-helix peptidic scaffolds. Peptides 2018, 107, 75-82. [CrossRef]

39. Imperial, J.S.; Bansal, P.S.; Alewood, P.F.; Daly, N.L.; Craik, D.J.; Sporning, A.; Terlau, H.; López-Vera, E.; Bandyopadhyay, P.K.; Olivera, B.M. A novel conotoxin inhibitor of Kv1. 6 channel and nAChR subtypes defines a new superfamily of conotoxins. Biochemistry 2006, 45, 8331-8340. [CrossRef]

40. Liu, Z.; Li, H.; Liu, N.; Wu, C.; Jiang, J.; Yue, J.; Jing, Y.; Dai, Q. Diversity and evolution of conotoxins in Conus virgo, Conus eburneus, Conus imperialis and Conus marmoreus from the South China Sea. Toxicon 2012, 60, 982-989. [CrossRef] [PubMed]

41. Robinson, S.D.; Safavi-Hemami, H.; McIntosh, L.D.; Purcell, A.W.; Norton, R.S.; Papenfuss, A.T. Diversity of Conotoxin Gene Superfamilies in the Venomous Snail, Conus victoriae. PLoS ONE 2014, 9, e87648.

42. Smith, J.J.; Undheim, E.A.B. True lies: Using proteomics to assess the accuracy of transcriptome-based venomics in centipedes uncovers false positives and reveals startling intraspecific variation in Scolopendra subspinipes. Toxins 2018, 10, 96. [CrossRef]

43. Endean, R.; Duchemin, C. The venom apparatus of Conus magus. Toxicon 1967, 4, 275-284. [CrossRef]

44. Matsui, T.; Hamako, J. Structure and function of snake venom toxins interacting with human von Willebrand factor. Toxicon 2005, 45, 1075-1087. [CrossRef]

45. Möller, C.; Davis, W.C.; Clark, E.; DeCaprio, A.; Marí, F. Conodipine-P1-3, the First Phospholipases A2 Characterized from Injected Cone Snail Venom. Mol. Cell. Proteom. 2019, 18, 876-891. [CrossRef]

46. de Teixeira, C.F.P.; Landucci, E.C.T.; Antunes, E.; Chacur, M.; Cury, Y. Inflammatory effects of snake venom myotoxic phospholipases A2. Toxicon 2003, 42, 947-962. [CrossRef]

47. Peiren, N.; de Graaf, D.C.; Vanrobaeys, F.; Danneels, E.L.; Devreese, B.; Van Beeumen, J.; Jacobs, F.J. Proteomic analysis of the honey bee worker venom gland focusing on the mechanisms of protection against tissue damage. Toxicon 2008, 52, 72-83. [CrossRef]

48. Cruz, L.J.; Ramilo, C.A.; Corpuz, G.P.; Olivera, B.M. Conus peptides: Phylogenetic range of biological activity. Biol. Bull. 1992, 183, 159-164. [CrossRef] [PubMed]

49. Vijayasarathy, M.; Basheer, S.M.; Balaram, P. Cone snail glutaminyl cyclase sequences from transcriptomic analysis and mass spectrometric characterization of two pyroglutamyl conotoxins. J. Proteome Res. 2018, 17, 2695-2703. [CrossRef]

50. Prator, C.A.; Murayama, K.M.; Schulz, R.J. Venom variation during prey capture by the cone snail, Conus textile. PLoS ONE 2014, 9, e98991. [CrossRef] [PubMed]

51. Dutertre, S.; Jin, A.H.; Kaas, Q.; Jones, A.; Alewood, P.F.; Lewis, R.J. Deep venomics reveals the mechanism for expanded peptide diversity in cone snail venom. Mol. Cell Proteom. 2013, 12, 312-329. [CrossRef] [PubMed]

52. Tucker, J.K.; Tenorio, M.J. Illustrated Catalog of the Living Cone Shells; MdM Publishing: Wincanton, UK, 2013.

53. Puillandre, N.; Duda, T.F.; Meyer, C.; Olivera, B.M.; Bouchet, P. One, four or 100 genera? A new classification of the cone snails. J. Molluscan Stud. 2015, 81, 1-23. [CrossRef] [PubMed]

54. Abalde, S.; Tenorio, M.J.; Uribe, J.E.; Zardoya, R. Conidae phylogenomics and evolution. Zool. Scr. 2019, 48, 194-214. [CrossRef] 
55. Abascal, F.; Zardoya, R.; Telford, M.J. TranslatorX: Multiple alignment of nucleotide sequences guided by amino acid translations. Nucleic Acids Res. 2010, 38, W7-W13. [CrossRef] [PubMed]

56. Katoh, K.; Standley, D.M. MAFFT multiple sequence alignment software version 7: Improvements in performance and usability. Mol. Biol. Evol. 2013, 30, 772-780. [CrossRef]

57. Lanfear, R.; Calcott, B.; Ho, S.Y.W.; Guindon, S. Partitionfinder: Combined selection of partitioning schemes and substitution models for phylogenetic analyses. Mol. Biol. Evol. 2012, 29, 1695-1701. [CrossRef] [PubMed]

58. Stamatakis, A. RAxML version 8: A tool for phylogenetic analysis and post-analysis of large phylogenies. Bioinformatics 2014, 30, 1312-1313. [CrossRef]

59. Andrews, S. FastQC: A Quality Control Tool for High Throughput Sequence Data. 2010. Available online: https://www. bioinformatics.babraham.ac.uk/projects/fastqc/ (accessed on 7 August 2021).

60. Grabherr, M.G.; Haas, B.J.; Yassour, M.; Levin, J.Z.; Thompson, D.A.; Amit, I.; Adiconis, X.; Fan, L.; Raychowdhury, R.; Zeng, Q.; et al. Full-length transcriptome assembly from RNA-Seq data without a reference genome. Nat. Biotechnol. 2011, 29, 644-652. [CrossRef] [PubMed]

61. Haas, B.J.; Papanicolau, A. TransDecoder. 2010. Available online: https://github.com/TransDecoder/TransDecoder (accessed on 7 August 2021).

62. Haas, B.J. Trinotate: Transcriptome Functional Annotation and Analysis. 2015. Available online: https://github.com/Trinotate/ Trinotate.github.io/wiki (accessed on 7 August 2021).

63. Camacho, C.; Coulouris, G.; Avagyan, V.; Ma, N.; Papadopoulos, J.; Bealer, K.; Madden, T.L. BLAST+: Architecture and applications. BMC Bioinform. 2009, 10, 421. [CrossRef] [PubMed]

64. Milne, I.; Stephen, G.; Bayer, M.; Cock, P.J.A.; Pritchard, L.; Cardle, L.; Shaw, P.D.; Marshall, D. Using Tablet for visual exploration of second-generation sequencing data. Brief. Bioinform. 2013, 14, 193-202. [CrossRef]

65. Langmead, B.; Salzberg, S.L. Fast gapped-read alignment with Bowtie 2. Nat. Methods 2012, 9, 357-359. [CrossRef]

66. Giribaldi, J.; Kazandjian, T.; Amorim, F.G.; Whiteley, G.; Wagstaff, S.C.; Cazals, G.; Enjalbal, C.; Quinton, L.; Casewell, N.R.; Dutertre, S. Venomics of the asp viper Vipera aspis aspis from France. J. Proteom. 2020, 218, 103707. [CrossRef]

67. Lavergne, V.; Harliwong, I.; Jones, A.; Miller, D.; Taft, R.; Alewood, P.F. Optimized deep-targeted proteotranscriptomic profiling reveals unexplored Conus toxin diversity and novel cysteine frameworks. Proc. Natl. Acad. Sci. USA 2015, 112, E3782-E3791. [CrossRef] [PubMed]

68. Lavergne, V.; Dutertre, S.; Jin, A.-H.; Lewis, R.J.; Taft, R.J.; Alewood, P.F. Systematic interrogation of the Conus marmoreus venom duct transcriptome with ConoSorter reveals 158 novel conotoxins and 13 new gene superfamilies. BMC Genom. 2013, 14, 708. [CrossRef] [PubMed]

69. Gao, B.; Peng, C.; Zhu, Y.; Sun, Y.; Zhao, T.; Huang, Y.; Shi, Q. High Throughput Identification of Novel Conotoxins from the Vermivorous Oak Cone Snail (Conus quercinus) by Transcriptome Sequencing. Int. J. Mol. Sci. 2018, 19, 3901. [CrossRef] [PubMed]

70. Team, R.C. R: A Language and Environment for Statistical Computing. 2013. Available online: https://www.r-project.org/ (accessed on 7 August 2021).

71. Pusev, R.; Gavrilov, I. normtest: Tests for Normality. 2014. Available online: https://cran.r-project.org/web/packages/normtest/ normtest.pdf (accessed on 7 August 2021).

72. Dinno, A. Nonparametric pairwise multiple comparisons in independent groups using Dunn's test. Stata J. 2015, 15, 292-300. [CrossRef]

73. Villanueva, R.A.M.; Chen, Z.J. ggplot2: Elegant Graphics for Data Analysis. 2019. Available online: https://ggplot2.tidyverse.org/ (accessed on 7 August 2021). 